\title{
Performance of Iterative DS-CDMA M-ary Demodulation in the Presence of Synchronization Errors
}

\author{
Pei Xiao, Erik Ström \\ Communication Systems Group, Dept. of Signals and Systems \\ Chalmers University of Technology, SE-412 96, Göteborg, Sweden \\ E-mail: pei.xiao@s2.chalmers.se, erik.strom@s2.chalmers.se
}

\begin{abstract}
The robustness of different interactive schemes for demodulating $M$-ary orthogonal signaling formats in asynchronous DS-CDMA systems to the synchronization errors is addressed in this paper. The system under study resembles the uplink of an IS-95 system. The channel is assumed to be a time-varing flat Rayleigh-fading channel. Our simulation results show that performance degradation for the considered multi-user detectors increase linearly with synchronization errors and eventually converge to that of conventional matched filter. In order to see the impact of channel estimation on the performance of multi-user detectors, we made some comparisons between non-coherent and coherent variants of the detection algorithms.
\end{abstract}

\section{Introduction}

The considered system in this paper is a DS-CDMA system with orthogonal modulation. The system resembles the uplink (reverse link) of an IS-95 system in that the narrowband bit stream is spread by one of $M$ possible Walsh codes, which are not used for separating users from each other, but for M-level modulation. Then the modulated data is scrambled with a long PN-code. Different scrambling codes are used to separate users.

Various M-ary demodulation schemes have been proposed by different authors. For instance, parallel and successive interference cancellation for $M$-ary orthogonal modulation in DS-CDMA are presented in [1], [2], [3]. The interferences are estimated and subtracted from the received signal before detection is done. Iterative schemes for demodulating $\mathrm{M}$-ary orthogonal signalling formats in DS-CDMA systems are proposed in [4], [5]. Interference suppression, i.e., removal of the multiple access interferences (MAI) by means of filtering (orthogonal projection), and interference cancellation, i.e., removal of MAI by means of subtraction, are studied. Time-varying Rayleigh fading channel is assumed in those paper, which entails the necessary channel estimation for effective interference cancellation and suppression.
The algorithms presented in the above papers assume perfect knowledge of the channel delay. In [7], Orten and Ottosson studied the impact of the synchroniztion errors on the system with BPSK modulation. However, an analysis of the system behavior under errors in delay estimates in DS-CDMA M-ary orthogonal signalling system is still lacking. This paper aims at investigating on the robustness of the different M-ary demodulation schemes against synchronization errors. As one would expect, the investigation results conclude that most of tested receivers are sensitive to synchronization errors with the exception of the conventional matched filter.

\section{System Model}

The passband received signal due to the $k$ th user is denoted by $r_{\mathrm{RF}, k}(t)$ and is formed as shown in the block diagram in Fig. 1. The $k$ th user's $j$ th symbol is denoted by $i_{k}(j) \in\{1,2, \ldots, M\}$, and the $M$ orthogonal signal alternatives are defined by $M$ Walsh sequences $\left\{w_{1}(n), w_{2}(n), \ldots, w_{M}(n)\right\}$ of length $N$. The Walsh chips are randomized by a scrambling code $c_{k}(n) \in\{-1,+1\}$. Hence, every symbol which represents $\log _{2} M$ binary bits is spread by $N$ chips and each bit by $N / \log _{2} M$ chips.

The baseband signal $s_{k}(t)$ is formed by pulse amplitude modulation with the unit-energy rectangular chip waveform $\psi(t)$, and the baseband signal is multiplied with a carrier with frequency $\omega_{c}$ and transmitted over the channel, which is represented by the complex channel gain $h_{k}(t)$ and assumed to be a slowly time-varying Rayleigh flat fading channel with delay $\tau_{k}$ and additive white Gaussian noise

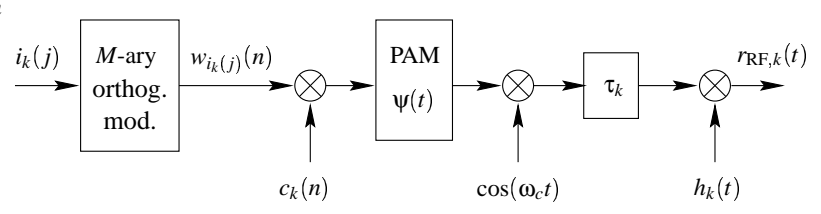

Figure 1. Block diagram of signal path for the $k$ th user 
The total received signal is the sum of the $K$ users' signals plus additive white complex Gaussian noise $n(t)$. The complex envelope of the received signal is

$$
r(t)=n(t)+\sum_{k=1}^{K} r_{k}(t), \quad r_{\mathrm{RF}, k}(t)=\sqrt{2} \operatorname{Re}\left\{r_{k}(t) e^{j \omega_{c} t}\right\}
$$

The received signal vector, $\mathbf{r}(j) \in \mathbb{C}^{N}$, due to transmission of the $j$ th symbol can be formed as

$$
\mathbf{r}(j)=\mathbf{A}(j) \mathbf{h}(j)+\mathbf{n}(j)=\sum_{k=1}^{K} \mathbf{a}_{h\lrcorner k}(j)+\mathbf{n}(j)
$$

The elements of $\mathbf{r}(j)$ are samples from the chip-matched filter. The zero-mean complex Gaussian random vector $\mathbf{n}(j) \in \mathbb{C}^{N}$ has second moments $\mathrm{E}\left[\mathbf{n}(j) \mathbf{n}^{T}(j)\right]=$ $\mathbf{0}$ and $\mathrm{E}\left[\mathbf{n}(j) \mathbf{n}^{*}(j)\right]=N_{0} \mathbf{I}_{N}$. The vector $\mathbf{h}(j) \in \mathbb{C}^{K}$ is defined by the complex channel gains as $\mathbf{h}(j)=$ $\left[h_{1}(j T) h_{2}(j T) \cdots h_{K}(j T)\right]$. The matrix $\mathbf{A}(j) \in \mathbb{R}^{N \times K}$ is defined as

$$
\begin{aligned}
\mathbf{A}(j) & =\left[\begin{array}{llll}
\mathbf{a}_{1}(j) & \mathbf{a}_{2}(j) & \cdots & \mathbf{a}_{K}(j)
\end{array}\right] \\
\mathbf{a}_{k}(j) & =\mathbf{C}_{k}(j) \mathbf{w}_{i_{k}(j)}
\end{aligned}
$$

where $\mathbf{C}_{k}(j) \in\{-1,+1\}^{N \times N}$ is a diagonal matrix defined by the $k$ th user's scrambling code, and $\mathbf{w}_{m}$ is the $m$ th column of the $N \times N$ Hadamard matrix.The noise-free received vector due to the $k$ th user's $j$ th symbol is denoted by $\mathbf{a}_{h \_k}(j)=\mathbf{a}_{k}(j) h_{k}(j)$.

Equation (1) is defined under the assumption of perfect synchronization. In case there is an error in the delay estimation at the receiver end, and assume the synchronization error is a fraction of chip rate duration $\tau_{e} / T_{c}\left(\tau_{e} \in\left[0, T_{c}\right]\right.$, $T_{c}$ is chip interval), the received vector should be reformed as:

$$
\begin{aligned}
\mathbf{r}(j)= & \left(\tau_{e} / T_{c}\right)\left[\sum_{k=1}^{K} \mathrm{us}\left(\mathbf{a}_{h \_k}(j), 1\right)+\sum_{k=1}^{K} \mathrm{ds}\left(\mathbf{a}_{h \_k}(j+1), N-1\right)\right] \\
& +\left(1-\tau_{e} / T_{c}\right) \sum_{k=1}^{K} \mathbf{a}_{h \_k}(j)+\mathbf{n}(j)
\end{aligned}
$$

where us(.), ds(.) stand for the up-shift and downshift operators respectively: $\quad$ us $\left(\left[\begin{array}{lll}a_{1} & \cdots & a_{N}\end{array}\right]^{T}, q\right)=$ $\left[\begin{array}{llllll}a_{N+1-q} & \cdots & a_{N} & 0 & \cdots & 0\end{array}\right]^{T}, \quad \operatorname{ds}\left(\left[\begin{array}{lll}a_{1} & \cdots & a_{N}\end{array}\right]^{T}, q\right)=$ $\left[\begin{array}{llllll}0 & \cdots & 0 & a_{1} & \cdots & a_{N-q}\end{array}\right]^{T}$.

For notation simplicity, all the above equations are derived for synchronous model in which $\tau_{k}=0$ for $k=$ $1,2, \ldots, K$. In asynchronous case, the vector $\mathbf{a}_{h \_k}(j)$ should be shifted accordingly based on the delay $\tau_{k}$ of each user.

\section{Receiver Algorithms}

The task of the receiver is to detect the symbols from all users given the received signal vector $\mathbf{r}(j)$, i.e., detect $i_{k}(j)$ for $k=1,2, \ldots, K$. For notation simplicity we will $\mathbf{a}(j)$, etc., whenever no ambiguity arises.

The decision on the $k$ th user's symbol at the $n$th iteration stage is denoted by $\hat{i}_{k}^{(n)}$ and is found as

$$
\hat{i}_{k}^{(n)}=\arg \max _{m \in\{1,2, \ldots, M\}} z_{k}^{(n)}(m)
$$

In the following, we shall briefly introduce how the soft decision $z_{k}(m)$ is obtained in different receiver algorithms.

\subsection{Conventional Matched Filter (Conv.)}

The conventional detection technique is to form the soft decision by correlating the received signal with the $M$ possible transmitted waveforms $\mathbf{a}_{k, 1}, \mathbf{a}_{k, 2}, \cdots, \mathbf{a}_{k, m}$. The soft decision can be formulated as $z_{k}(m)=\left|\mathbf{w}_{m}^{*} \mathbf{C}_{k}^{*} \mathbf{r}\right|=\left|\mathbf{a}_{k, m}^{*} \mathbf{r}\right|$ where we defined $\mathbf{a}_{k, m}=\mathbf{C}_{k} \mathbf{w}_{m}$. This simple scheme is particularly useful in the beginning of the detection process e.g. at the first iteration stage, when the estimates of the fading channel are lacking, we must therefore carry out the detection in a noncoherent manner.

\subsection{Whitened Matched Filter (WMF)}

As we know, the conventional matched filter achieves the best performance in the AWGN single user channel or in strict orthogonal synchronous channel. It's not a good choice for multiuser detection in which MAI must be taken into account in addition to the white Gaussian noise. MAI combined with Gaussian noise no longer has Gaussian distribution. A way to work around this problem is to whiten the combined noise, which can be achieved by preprocessing the received vector $\mathbf{r}$ with the matrix $\mathbf{R}^{-1 / 2} . \mathbf{R}^{-1 / 2}$ is obtained from $\mathbf{R}$ which is the correlation matrix for $\mathbf{r}$ by Cholesky factorization [6]. And $\mathbf{R}$ can be calculated as

$$
\mathbf{R}=\mathrm{E}\left[\mathbf{r r}^{*}\right]=\mathbf{A} \mathrm{E}\left[\mathbf{h h}^{*}\right] \mathbf{A}^{*}+N_{0} \mathbf{I}_{N}=\mathbf{A} \mathbf{P} \mathbf{A}^{*}+N_{0} \mathbf{I}_{N}
$$

The noncoherent WMF receiver can be formulated as

$$
z_{k}^{(n)}(m)=\frac{\left|\left(\hat{\mathbf{R}}^{-1 / 2} \mathbf{a}_{k, m}\right)^{*} \hat{\mathbf{R}}^{-1 / 2} \mathbf{r}\right|}{\left\|\hat{\mathbf{R}}^{-1 / 2} \mathbf{a}_{k, m}\right\|^{2}}=\frac{\left|\mathbf{a}_{k, m}^{*} \hat{\mathbf{R}}^{-1} \mathbf{r}\right|}{\mathbf{a}_{k, m}^{*} \hat{\mathbf{R}}^{-1} \mathbf{a}_{k, m}}
$$

where $\hat{\mathbf{A}}$ is obtained by substituting $\hat{i}_{k}^{(n-1)}$ into (2). $\hat{\mathbf{R}}$ is the estimate of $\mathbf{R}$ based on data estimate $\hat{\mathbf{A}}$. The numerator represents the signal power at the matched filter output for the candidate vector $\left\{\hat{\mathbf{R}}^{-1 / 2} \mathbf{a}_{k, m}\right\}$. The denominator stands for the whitened noise energy. We choose the transmitted waveform $\mathbf{a}_{k, m}$ in such a way that it maximizes the SNR expressed by (5).

Its coherent variant is found by incorporating the channel estimate $\hat{h}_{k}$ in the above soft decision function:

$$
z_{k}^{(n)}(m)=\frac{\operatorname{Re}\left\{\hat{h}_{k}^{*} \mathbf{a}_{k, m}^{*} \hat{\mathbf{R}}^{-1} \mathbf{r}\right\}}{\mathbf{a}_{k, m}^{*} \hat{\mathbf{R}}^{-1} \mathbf{a}_{k, m}}
$$


formance of noncoherent WMF against its coherent counterpart. In the simulations, each user transmits one of $M=8$ Walsh codes spread to a total length of $N=32$ chips. The scrambling codes $c_{k}(n)$ are random. The signal to noise ratio is set to $10 \log _{10} E_{b} / N_{0}=25 \mathrm{~dB}$. With conventional noncoherent initial stage, $L=10$ iteration stages are performed on both receiver algorithms. The same parameter setting applies to the subsequent experiments. Also the perfect power control is assumed except in the near-far resistance testing conducted in Section 5. As illustrated in Fig. 5, smoothed coherent $\mathrm{WMF}^{1}$ gives better performance than noncoherent WMF. Although only WMF algorithm is tested, this is generally true provided that the complex channel gains are accurately estimated.

Note that the coherent version of the receiver requires estimates of the fading processes, i.e., an estimate of $h_{k}$. The algorithms for estimating the fading are presented in Section 4.

\subsection{Parallel Interference Cancellation (PIC)}

The basic principle underlying the interference cancellation algorithms is to subtract the detected user signals from the received waveform so that it is less contaminated for the user of interest when we do the demodulation for that particular user. The subtractive interference cancellation can be done in sequence or in parallel. The successive interference cancellation (SIC) is suitable for systems in which the powers of the signals from different users vary in a wide range. However, parallel interference cancellation (PIC) seems to perform better in this considered system in which equal power is imposed on different users. We can remove all the interfering users' contributions to $\mathbf{r}$ before detection, which would hopefully lead to better data estimates and performance may be further improved by repeating the process in an iterative manner. We initialize the iteration with a conventional noncoherent stage. For subsequent stages $n=2,3, \ldots, N_{i}$, we iterate

$$
z_{k}^{(n)}(m)=\operatorname{Re}\left\{\hat{h}_{k}^{*} \mathbf{a}_{k, m}^{*}\left[\mathbf{r}-\hat{\mathbf{A}} \hat{\mathbf{h}}+\hat{h}_{k} \hat{\mathbf{a}}_{k}\right]\right\}
$$

where $\hat{\mathbf{A}}$ and $\hat{\mathbf{a}}_{k}$ are obtained by substituting $\hat{i}_{k}^{(n-1)}$ into (2). $\hat{\mathbf{h}}$ is the estimate of the fading vector $\mathbf{h}$.

\subsection{Iterative Interference Suppression (IIS)}

IIS differs from PIC in that it suppresses the MAI via orthogonal projection rather than subtraction.

If we delete the column due to the $k$ th user from the matrix $\mathbf{A}$ and form the matrix $\mathbf{U}$ :

$$
\mathbf{U}=\left[\begin{array}{lllllll}
\mathbf{a}_{1} & \mathbf{a}_{2} & \cdots & \mathbf{a}_{k-1} & \mathbf{a}_{k+1} & \cdots & \mathbf{a}_{K}
\end{array}\right]^{T}
$$

\footnotetext{
${ }^{1}$ There are two variations of coherent detection. Namely, smoothed and unsmoothed coherent detection, which represents two way of estimating the channel gains, as will be discussed later.
}

onto the orthogonal complement to the subspace spanned by the columns of $\mathbf{U}$. $\mathbf{U}^{\dagger}$ denotes the left psuedoinverse of $\mathbf{U}$. This implies that $\mathbf{P}_{\mathbf{U}}^{\perp} \mathbf{a}_{i}=\mathbf{0}$ for all $i \neq k$, and thus the interferences are suppressed by projecting $\mathbf{r}$ on $\mathbf{P}_{\mathbf{U}}^{\perp}$ :

$$
\mathbf{P}_{\mathbf{U}}^{\perp} \mathbf{r}=\mathbf{P}_{\mathbf{U}}^{\perp}[\mathbf{A h}+\mathbf{n}]=\sum_{i=1}^{k} \mathbf{P}_{\mathbf{U}}^{\perp} \mathbf{a}_{i} h_{i}+\mathbf{P}_{\mathbf{U}}^{\perp} \mathbf{n}=\mathbf{P}_{\mathbf{U}}^{\perp} \mathbf{a}_{k} h_{k}+\mathbf{P}_{\mathbf{U}}^{\perp} \mathbf{n}
$$

This leads to the IIS detection algorithm (coherent version):

$$
z_{k}^{(n)}(m)=\operatorname{Re}\left\{\hat{h}_{k}^{*} \mathbf{a}_{k, m}^{*} \mathbf{P}_{\hat{\mathbf{U}}}^{\perp} \mathbf{r}\right\}-0.5\left\|\mathbf{P}_{\hat{\mathbf{U}}}^{\perp} \mathbf{a}_{k, m} \hat{h}_{k}\right\|^{2}
$$

where $\hat{\mathbf{U}}$ is the estimate of $\mathbf{U}$, i.e., the detected interferences at the last iteration stage.

\section{Channel Estimation}

Recall from (1) that $\mathbf{r}=\mathbf{A h}+\mathbf{n}$. By using the estimate of the transmitted data during the $j$ th symbol interval $\hat{\mathbf{A}}(j)$, and neglecting the noise term $\mathbf{n}$, we can estimate $\mathbf{h}(j)$ as

$$
\hat{\mathbf{h}}(j)=\hat{\mathbf{A}}^{\dagger}(j) \mathbf{r}(j)
$$

where $\hat{\mathbf{A}}^{\dagger}$ denotes the left psuedoinverse of $\hat{\mathbf{A}}$.

Another alternative is the linear minimum mean square estimate of $\mathbf{h}: \hat{\mathbf{h}}=\hat{\mathbf{p}}^{*} \hat{\mathbf{R}}^{-1} \mathbf{r}$, where $\hat{\mathbf{R}}$ is the correlation matrix for $\hat{\mathbf{r}}$ as introduced above, and $\hat{\mathbf{p}}$ is the crosscorrelation matrix between $\mathbf{r}$ and $\hat{\mathbf{h}}: \hat{\mathbf{p}}=\mathrm{E}\left[\mathbf{r} \hat{\mathbf{h}}^{*}\right]=\hat{\mathbf{A}} \mathbf{P}$.

The algorithms are decision-directed, that is, the channel estimation in the $l$ th iteration stage uses the data estimates from the previous stage, i.e., $\hat{i}_{k}^{(l-1)}$. Fig. 2 illustrates the original fading channel of a single user, Fig. 3 shows the results of channel estimation using the approach expressed by (9). Corresponding plot for the MMSE alogrithm is very similar and thus omitted to conserve space. Unfortunately, the estimates are noisy. We know that the channel gains are correlated in time, and we should therefore be able to improve the estimates by smoothing. A simple smoothing procedure is to feed $\hat{\mathbf{h}}(j)$ through an FIR filter. Using Hamming window of length 19 , the channel estimation results after smoothing operation are shown in Fig. 4. It is very close the original channel.

Fig. 5 illustrates the impact of channel estimation and smoothing operation on the performance of receiver algorithms. Here again we use WMF as an example, it's evident that coherent detection with smoothed channel estimates achieves the best results.

\section{Robustness of Multiuser Detectors to Syn- chronization Errors}

Synchronization is a difficult task in DS-CDMA systems. Due to interference and noise, timing cannot be exactly estimated and synchronization error will therefore exist. Thus, it is of interest to investigate the effect of imperfect timing estimation on the different algorithms. To 
